\title{
On a Quasi-Variational Inequality Arising in Semiconductor Theory
}

\author{
JoSÉ-FRANCISCO RODRIGUES(*)
}

\begin{abstract}
Some new mathematical results of existence and uniqueness of solutions are obtained for a class of quasi-variational inequalites modelling the free boundary problem for the determination of the depletion zone in reverse biased semiconductor diodes. The corresponding one (or two) obstacle implicite problems are solved by direct methods with weak regularity estimates for mixed boundary value elliptic problems of second order.
\end{abstract}

\section{INTRODUCTION}

The van Roosbroek's model for semiconductor devices consists of an interesting nonlinear diffusion system of equations which has been widely studied in recent years (see, for instance, [MRS] and its references).

For the steady-state case of a $p n$-junction diode under strong reverse bias, after a singular perturbation analysis, the determination of the depletion layer leads to a free boundary problem. For this approximating problem, a double obstacle variational inequality has been proposed for the electrostatic potential $u=u(x)$, which is supposed to be defined for $x \in \Omega \subset \mathbb{R}^{N}$, where $\Omega$ is a bounded domain representing the semiconductor part of an electronic device (see $[\mathrm{HN}],[\mathrm{BCM}]$, [S] or [MRS]).

This limit problem consists of finding $u$, such that,

$$
\begin{array}{ll}
\psi \leq u \leq \varphi & \text { in } \Omega, \text { and } \\
-\Delta u=f & \text { in the region } D=\{\psi<u<\varphi\}
\end{array}
$$

* Work done in the framework of research project JNICT/87046.

1991 Mathematics Subject Classification: 35R35, 35J85, 78A30

Editorial Complutense. Madrid, 1992. 
with continuity conditions on the free boundaries $\partial\{u=\varphi\}$ and $\partial\{u=\psi\}$ and with mixed Dirichlet-Neumann conditions on the fixed boundary $\partial \Omega$.

In the case of total depletion assumption, $\varphi$ and $\psi$ are given constants corresponding to the constant values of the potential at the neutral regions, which are considered then as being fully conducting. In the noncoincidence set $D$, also called the depletion zone, the potential distribution is governed by the Poisson equation (1.2), where $f$ models the doping effects. For a singular perturbation analysis of this problem, see [BCM], [BCG] or [Ga] for the onedimensional case, $[\mathrm{CF}]$ for the bidimensional problem with simplified boundary conditions, and [HN], [S] for a discussion of this formal limit problem.

A more complex asymptotic model has been proposed in [NM], where the physical parameters $\varphi=\varphi(x)$ and $\psi=\psi(x)$ are the socalled Fermi quasipotentials, which are functions depending implicitly on the potential $u$. Actually, in [NM], the domain $\Omega$ is of the form $\Omega=\Omega_{1} \cup \Gamma \cup \Omega_{2}$, where the $p n$-junction $\Gamma$, given by a smooth known interface, separates two simply connected subdomains $\Omega_{1}$ and $\Omega_{2}$. The first one, $\Omega_{1}$, is dominated by the contributions from the negatively charged free electrons (with density $n=n(x)$ ), while the second one, $\Omega_{2}$, by the positively charged holes (with density $p=p(x)$ ).

Under certain simplificating assumptions, in particular, neglecting respectively, in $\Omega_{2}$ and $\Omega_{1}$, the densities

$$
n=n_{1} \exp \left[-k_{1}(u-\varphi)\right] \text { and } p=n_{2} \exp \left[-k_{2}(u-\psi)\right]
$$

they may be considered defined only in the subregions $\Omega_{1}$ and $\Omega_{2}$, respectively. Here $n_{1}, n_{2}, k_{2}=-k_{\mathrm{I}}=k>0$ are known physical constants of the model. Then, following [NM], the bilateral condition may be replaced by

$$
u \leq \varphi \text { in } \Omega_{1} \text { and } u \geq \psi \text { in } \Omega_{2},
$$

and in the depletion zone we have

$$
-\Delta u=f_{1} \text { in }\{u<\varphi\} \cap \Omega_{1} \text { and }-\Delta u=f_{2} \text { in }\{u>\psi\} \cap \Omega_{2} .
$$

The relation between $u$ and $\varphi, \psi$ is given by a nonlinear operator $u \rightarrow\{\Phi(u), \Psi(u)\}$, which is defined by logarithmic transformations of the solutions $w_{1}$ and $w_{2}$ of the following mixed boundary value problems in $\Omega_{1}$ and $\Omega_{2}$, respectively, for $i=1,2$ :

$$
\begin{array}{ll}
\nabla \cdot\left(e^{-k_{i}{ }^{2}} \nabla w_{i}\right)=0 & \text { in } \Omega_{i}, \\
w_{i}=e^{k_{i} g_{i}} \text { on } \quad \Gamma_{i}, & \partial w_{i} / \partial n=0 \quad \text { on } \partial \Omega_{i} \backslash \Gamma_{i} .
\end{array}
$$


Here $\Gamma_{i} \subset \partial \Omega_{i}$ is an open subset of the boundary $\partial \Omega_{i}$, where the values of $g_{i}$ are prescribed in a compatible way with the reverse biased conditions.

The equations (1.6) for $w_{1}$ and $w_{2}$ are derived from the steady-state drift diffusion equations for the negative and positive carrier concentrations, respectively $n$ and $p$. Using the relations (1.3), the Fermi quasi-potentials are then given, respectively, by

$$
\varphi=\frac{1}{k_{1}} \log w_{1}=\Phi(u) \text { and } \psi=\frac{1}{k_{2}} \log w_{2}=\Psi(u) .
$$

This general formulation, with the Fermi quasi-potentials as obstacles in two disjoint subdomains, may be decoupled into two model problems, for mathematical or approximating purposes, as suggested in [NM] or [M]. For instance, taking into account only the effects of carriers of type $p$, we shall consider first the following implicit unilateral problem in $\Omega=\Omega_{2}$.

$$
u \geq \Psi(u),-\Delta u \geq f \text { and }(-\Delta u-f)(u-\Psi(u))=0 \text { a.e. in } \Omega
$$

where the obstacle $\Psi$ is defined by (1.8) and by the solution $w_{2}$ of (1.6)-(1.7). To complete this formulation we need to add, for instance, a mixed boundary condition of the following type $\left(\Gamma_{o} \subset \partial \Omega, \Gamma_{o} \neq \varnothing\right)$ :

$$
u=h \quad \text { on } \quad \Gamma_{o} \quad \text { and } \quad \partial u / \partial n=0 \quad \text { on } \quad \partial \Omega \backslash \Gamma_{o} \text {. }
$$

By applying general results on quasi-variational inequalities (see [M], $[\mathrm{BC}],[\mathrm{BL}]$, for instance) and using restrictive estimates on $\nabla u$ in $L^{\infty}$, the onedimensional problem (1.9) and a particular two-dimensional case, with small data, has been considered by Nassif in [N]. Using a direct and, in this case, better approach, which is based on the properties of the obstacle problem (see, e.g., [KS] and [R]) we are able, in Section 2, to solve (1.9)-(1.10) with general assumptions and without any restriction on the space dimension. In Section 3, we discuss sufficient conditions for the uniqueness of the solution with small data, improving the results of [N]. Finally, in Section 4, we extend our results to the model with two obstacles, corresponding to the pnjunctions case.

\section{EXISTENCE OF A SOLUTION TO THE QUASI-VARIATIONAL INEQUALITY}

In this section we let $\Omega$ be a bounded domain of $\boldsymbol{R}^{N}$, for arbitrary $N \geq 1$ and with Lipschitz boundary $\partial \Omega$ if $\mathrm{N} \geq 2$. We consider the quasi-variational 
inequality (1.9) which, incorporating the mixed boundary conditions (1.10), has the form:

$$
u \in \mathbf{K}(u) ; \quad \int_{\Omega} \nabla u \cdot \nabla(v-u) d x \geq \int_{\Omega} f(v-u) d x, \quad \forall v \in \mathbf{K}(u) .
$$

Here the convex set $\mathbf{K}(u)$ depends on the solution itself through

$$
\mathbf{K}(z)=\left\{v \in H^{1}(\Omega): v=h \text { on } \Gamma_{0} \text { and } v \geq \Psi(z) \text { in } \Omega\right\} \text {, }
$$

where, for an arbitrary $z \in \mathrm{L}^{\infty}(\Omega)$, the solution $w=w(z)$ of the following mixed elliptic problem

$$
\begin{aligned}
& \nabla \cdot\left(e^{-k z} \nabla w\right)=0 \text { in } \Omega, \\
& w=e^{k g} \quad \text { on } \quad \Gamma_{D} \quad \text { and } \partial w / \partial n=0 \text { on } \partial \Omega \backslash \Gamma_{D},
\end{aligned}
$$

defines the obstacle of (2.2) by the relation

$$
\Psi(z)=\frac{1}{k} \log w(z)
$$

We assume $k>0$ is a given constant, $\Gamma_{o}$ and $\Gamma_{D}$ are regular, non-empty, open subsets of the boundary $\partial \Omega$, and $f, g$ are given functions, such that

$$
\begin{aligned}
& f \in L^{p / 2}(\Omega) \text { and } g, h \in W^{1-1 / p, p}(\partial \Omega) \text { for some } p>\mathrm{N}>1, \text { and } \\
& f \in L^{1}(\Omega) \text { and } g, h \text { take constant values, for } \mathrm{N}=1 .
\end{aligned}
$$

Lemma 2.1 For any $z \in L^{\infty}(\Omega)$, there exists a unique $w=w(z) \in$ $H^{1}(\Omega) \cap C^{0, \alpha}(\bar{\Omega})$, for some $0<\alpha \leq 1-N / p$, solving (2.3)-(2.4). Moreover $w$ satisfies the estimates

$$
\begin{gathered}
0<e^{k \eta_{*}} \equiv \inf _{\Gamma_{D}} e^{k g} \leq w \leq e^{k \eta^{*}} \equiv \sup _{\Gamma_{D}} e^{k g} \text { in } \Omega, \\
\left\|w\left(z_{1}\right)-w\left(z_{2}\right)\right\|_{H^{\prime}(\Omega)} \leq C\left\|z_{1}-z_{2}\right\|_{L^{\infty}(\Omega)}
\end{gathered}
$$

where $C>0$ depends on $\left\|z_{1}\right\|_{\mathrm{L}^{\infty}(\Omega)}$ and $\left\|z_{2}\right\|_{\mathrm{L}^{\infty}(\Omega)}$.

Proof: Noting that, for $z \in L^{\infty}(\Omega)$, we have

$$
0<\varsigma_{*}=\inf _{\Omega} e^{-k z} \leq e^{-k z} \leq \varsigma^{*}=\sup _{\Omega} e^{-k z} \text { in } \Omega,
$$


The first part of this lemma is immediate by the elliptic theory, the Holderian estimates up to the boundary (note that $g \in C^{0,1-\mathrm{N} / p}(\partial \Omega)$ ) and the maximum principle.

To prove (2.8) we write (2.3)-(2.4) in variational form for $w_{i}=w\left(z_{i}\right)$ :

$$
\int_{\Omega} e^{-k z_{i}} \nabla w_{i} \cdot \nabla v d x=0, \quad \forall v \in H^{\prime}(\Omega): v \mid \Gamma_{D}=0, \text { for } \ddot{i}=1,2,
$$

and we take $v=w_{1}-w_{2} \equiv \bar{w}$; letting $\varsigma_{1}=\underset{\Omega}{\inf } e^{-k z_{1}}>0$, we obtain

$$
\begin{gathered}
\zeta_{1}\|\nabla \bar{w}\|_{L^{2}(\Omega)}^{2} \leq \int_{\Omega} e^{-k z_{1}}|\nabla \bar{w}|^{2} d x=\int_{\Omega}\left(e^{-k z_{2}}-e^{\left.-k z_{1}\right)}\right) \nabla w_{2} \cdot \nabla \bar{w} d x \\
\leq\left\|e^{-k z_{1}}-e^{-k z_{2}}\right\|_{L^{\infty}(\Omega)}\left\|\nabla w_{2}\right\|_{L^{2}(\Omega)}\|\nabla \bar{w}\|_{L^{2}(\Omega)} .
\end{gathered}
$$

Since $\left\|\nabla w_{2}\right\|_{L^{2}(\Omega)}$ is bounded by some constant depending on $\left\|z_{2}\right\|_{L_{\infty}^{\infty}(\Omega)}$ and on $\|g\|_{\mathrm{H}^{1 / 2}(\partial \Omega)}$, from (2.9) we easily obtain the estimate (2.8), recalling the Poincaré inequality for $\bar{w}=w\left(z_{1}\right)-w\left(z_{2}\right)$.

Remark 2.1 The global estimates of DeGiogi-Stampacchia imply that the bound on $\|w\|_{C^{0, a}(\bar{\Omega})}$ depends only on the constants $\varsigma_{*}, \zeta^{*}$ and the Dirichlet data $g$ (see, e.g., [R], page 170 , and its references). Hence as an immediate consequence of (2.8), the nonlinear mapping $z \rightarrow w(z)$ is sequentially continuous from $\mathrm{L}^{\infty}(\Omega)$ into $\mathrm{H}^{\mathrm{t}}(\Omega) \cap \mathrm{C}^{0, \alpha^{\prime}}(\bar{\Omega})$ for any $0 \leq \alpha^{\prime}<\alpha$, for the strong topologies.

As an immediate consequence of Lemma 2.1 and Remark 2.1, we can state:

Lemma 2.2 For any $z \in L^{\infty}(\Omega)$, the obstacle $\Psi=\Psi(z)=\frac{1}{k} \log w(z)$ is, such that, $\Psi \in \mathrm{H}^{1}(\Omega) \cap \mathrm{C}^{0, \alpha}(\bar{\Omega})$, for some $0<\alpha \leq 1-\mathrm{N} / p, \Psi=g$ on $\Gamma_{D}$ and

$$
\eta * \leq \Psi \leq \eta^{*} \text { in } \Omega \text {, independently of } z \in \mathrm{L}^{\infty}(\Omega)
$$

In addition, the mapping $\Psi: z \rightarrow \Psi(z)$ is sequentially continuous from $\mathrm{L}^{\infty}(\Omega)$ into $\mathrm{H}^{\prime}(\Omega) \cap \mathrm{C}^{0, \alpha^{\prime}}(\bar{\Omega})$ for any $0 \leq \alpha^{\prime}<\alpha$, i.e., if $z_{n} \rightarrow z$ in $\mathrm{L}^{\infty}(\Omega)$ then $\Psi\left(z_{n}\right) \rightarrow \Psi(z)$ in $\mathrm{H}^{\prime}(\Omega) \cap \mathrm{C}^{0, \alpha^{\prime}}(\bar{\Omega})$. 
$\therefore$ In order to solve (2.1) we consider, for any $z \in L^{\infty}(\Omega)$, the auxiliary obstacle problem

$$
u_{z} \in \mathbf{K}(z): \int_{\Omega} \nabla u_{z} \cdot \nabla\left(v-u_{z}\right) d x \geq \int_{\Omega} f\left(v-u_{z}\right) d x, \quad \forall v \in \mathbf{K}(z)
$$

and we recall some well-known properties of the translated problem:

$$
\tilde{u} \in \mathbf{K}_{\tilde{\psi}}: \int_{\Omega} \nabla \tilde{u} \cdot \nabla(v-\tilde{u}) d x \geq 0, \quad \forall v \in \mathbf{K}_{\tilde{\psi}},
$$

Here, if we suppose $\tilde{\psi} \in H^{\prime}(\Omega)$, such that, $\tilde{\psi} \leq 0$ on $\Gamma_{o}$, we have

$$
\mathbf{K}_{\tilde{\psi}}=\left\{v \in \mathbf{H}^{1}(\Omega): v=0 \text { on } \Gamma_{v} \text { and } v \geq \tilde{\psi} \text { in } \Omega\right\} \neq \varnothing \text {. }
$$

Using the standard notations $\Lambda$ and $V$ for inf and sup, respectively, we recall the following results:

Proposition 2.1 For each $\tilde{\psi} \in \mathrm{H}^{\prime}(\Omega) \cap \mathrm{L}^{\infty}(\Omega), \tilde{\psi} \leq 0$ on $\Gamma_{b}$, there exists a unique solution $\tilde{u}$ of (2.12) verifying the estimate

$$
0 \leq \tilde{u} \leq 0 \vee \sup \tilde{\psi} \quad \text { in } \Omega .
$$

Moreover the corresponding mapping $\tilde{\psi} \rightarrow \tilde{u}(\tilde{\psi})$ is sequentially continuous from $H^{I}(\Omega)$ into $H^{t} .(\Omega)$ and a contraction in $\mathrm{L}^{\infty}(\Omega)$ :

$$
\left\|\tilde{u}\left(\psi_{1}\right)-\tilde{u}\left(\psi_{2}\right)\right\|_{1} \infty(\Omega) \leq\left\|\psi_{1}-\psi_{2}\right\|_{1} \infty(\Omega)
$$

Proof: See, e.g., [R], Chap 4.

The reduction of the problem (2.11) to (2.12) is'done by considering the mixed linear problem:

$$
-\Delta \xi=f \text { in } \Omega, \xi=h \text { on } \Gamma_{o} \text { and } \partial \xi / \partial n=0 \text { on } \partial \Omega \backslash \Gamma_{\sigma} .
$$

By $\mathrm{C}^{0 . \alpha}$-estimates, we know that the unique solution of (2.16) satisfies, for some constant $\mathrm{C}>0$ and some $0<\alpha \leq 1-\mathrm{N} / p$ (recall (2.6)):

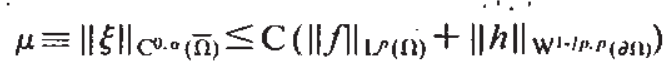


Then, letting $\tilde{u}=u_{z}-\xi$ and $\tilde{\psi}=\Psi(z)-\xi$ we establish the equivalence between (2.11) and (2.12), for every fixed $z \in L^{\infty}(\Omega)$, such that, $K(z) \neq \varnothing$. This compatibility condition can be guaranteed, for instance, by assuming

$$
\underset{\Gamma_{a} \Gamma_{D}}{\inf } h \geq \sup _{\Gamma_{D}} g \text { and } g \leq h \text { on } \Gamma_{O} \cap \Gamma_{D}
$$

Lemma 2.3. Assuming (2.6) and (2.18), for any $z \in L^{\infty}(\Omega)$, there exists a unique $u_{z} \in \mathbf{K}(z) \cap C^{0, \gamma}(\bar{\Omega})$, for some $0<\gamma<1$, solving (2.II) which satisfies the estimate

$$
\eta_{*} \leq u_{z} \leq 2 \mu+\eta^{*} \quad \text { in } \Omega, \text { independently of } z
$$

Moreover, the mapping $z \rightarrow u_{z}$ is sequentially continuous from $L^{\infty}(\Omega)$, into $H^{\prime}(\Omega) \cap C^{\prime} \cdot \gamma^{\prime}(\bar{\Omega}), 0 \leq \gamma^{\prime}<\gamma$, and it satisfies

$$
\left\|u_{z}-u_{i}\right\|_{c^{\circ}(\bar{\Omega})} \leq\|\Psi(z)-\Psi(\hat{z})\|_{C(\bar{\Omega})} .
$$

Proof: Due to the equivalence of (2.11) and (2.12), we need to garantee the admissibility condition $\mathbf{K}(z) \neq \varnothing$, i.e.,

$$
\tilde{\psi}=\Psi(z)-h \leq 0 \quad \text { on } \Gamma_{o} \text {, independently of } z \in \mathrm{L}^{\infty}(\Omega) .
$$

Since $\Psi(z)=g$ on $\Gamma_{D}$, of course that condition is allways verified if $\Gamma_{o} \subset \Gamma_{D}$, but in general, we only know that, in $\bar{\Omega}$,

$$
\underset{\Gamma_{D}}{\inf } g=\eta * \leq \Psi(z) \leq \eta^{*}=\underset{\Gamma_{D}}{\sup } g, \text { for all } z \in \mathrm{L}^{\infty}(\Omega)
$$

and this is the reason to require the sufficient condition (2.18).

Recalling the Hölder continuity of the solutions of the obstacle problem (see [R], Section 5:7) and the Proposition 2.1, we complete the proof of this lemma by noting that for $u_{z}=\tilde{u}+\xi$ we have:

$$
\Psi(z) \leq u_{z} \leq \xi+\sup _{\Omega}[\Psi(z)-\xi]^{+} .
$$

Remark 2.2 The assumption (2.18) is satisfied in the physical situation considered in [N], where $\Gamma_{D} \subset \Gamma_{o}$ and $g$ traduces the reverse-biased conditions $\left(g>0\right.$ on $\Gamma_{D} \backslash \Gamma_{o}$ and $g \leq 0$ on $\left.\Gamma_{D}\right)$. Notice that, in [N], $u$ and $g$ were considered with the opposite signs, corresponding to an upper obstacle, as for the case of carriers of type $n$ only. 
Theorem 2.1 Under the assumptions (2.6) and (2.18), there exists at least a solution $u$ to the quasi-variational inequality (2.1). Moreover, $u$ has the regularity

$$
u \in \mathrm{C}^{0, \gamma}(\bar{\Omega}) \cap \mathrm{W}_{\mathrm{l}, \infty}^{2, \infty}(\Omega), \text { for some } 0<\gamma<1
$$

Proof: The solution $u$ is given by any fixed point of the mapping $T$ : $z \rightarrow u_{z}$, defined by the auxiliary problem (2.11). Using the "a priori" estimate (2.19), we consider as domain of $T$ the convex set:

$$
\mathbf{M}=\left\{v \in \mathbf{C}^{\circ}(\bar{\Omega}): \eta * \leq v \leq 2 \mu+\eta^{*} \text { in } \Omega\right\}
$$

of the Banach space $\mathrm{C}^{0}(\bar{\Omega})$. Since Lemma 2.3 yields $T(\mathbf{M}) \subset \mathbf{M} \cap \mathrm{C}^{0, \gamma}(\bar{\Omega})$, by the compactness of $\mathrm{C}^{0, \gamma}(\bar{\Omega}) \subset \mathrm{C}^{\circ}(\bar{\Omega})$, the Schauder. fixed point theorem guarantees the existence of $u=T u$.

Since $u \in C^{0 . \gamma}(\bar{\Omega})$, by local regularity of the solution of the equation (2.3), we find $w(u) \in C^{l, \gamma}(\Omega)$ and then also $\Psi(u) \in C^{l} \cdot \gamma(\Omega)$. Hence, by the local regularity of the obstacle problem, we have also $u \in C^{1, \gamma}(\Omega)$ and, by iterating once, it follows $w(u)$ and $\Psi(u)$ in $\mathrm{C}^{2}, \gamma(\Omega)$, which is then sufficient to obtain the optimal regularity $u \in W_{\text {loc }}^{2, \infty}(\Omega)$ (see, e.g., [R]).

Remark 2.3 This existence result considerably extends [N], which only covered the cases $N=1$ and $N=2$ with piecewise constant $g$, with $0 \leq f \in L^{\infty}(\Omega)$ and with a very restritive smallness condition on $g$. This was due to the method of [N] that required an "a priori" estimate on $\|\left.\nabla W^{*}\right|_{L^{*}(\Omega)}$, which in general does not hold for the mixed problem (2.3)-(2.4).

\section{UNIQUENESS OF SOLUTIONS FOR SMALL DATA}

The existence of a solution in the preceding section does not require any restriction on the size of the data, since it was based on the Schauder fixed point theorem. For the same mapping $T: z \rightarrow u_{z}$, we investigate now sufficient conditions in order to make $T$ a strict contraction in the metric space $M$, defined in (2.22). This will imply the uniqueness of the solution, by the Banach fixed point theorem. It turns out, that it is sufficient to improve the Lipschitz dependence (2.8) for the solution $w$ of the mixed boundary value problem (2.3)-(2.4), with respect to $z$.

For $p>N$ and if $\Gamma_{D} C \partial \Omega$ has positive (N-1)-measure, we recall that, by the Poincaré and Sobolev inequalities, we have

$$
\|v\|_{C^{0}(\bar{\Omega})} \leq C_{p}\|\nabla v\|_{L^{r}(\Omega)} \quad \text { for all } v \in W_{\Gamma_{b}}^{1, p}(\Omega)
$$


where $C_{p}=C\left(p, N, \Omega, \Gamma_{D}\right)>0$ is a fixed constant, and $\mathrm{W}_{\Gamma_{p}, p}^{l, p}(\Omega)$ is the closure of the set $\left\{v \in \mathrm{C}^{\prime}(\bar{\Omega})\right.$ : supp $\left.v \cap \widetilde{\Gamma}_{D}=\varnothing\right\}$ in the Sobolev space $\mathrm{W}^{1, \rho}(\Omega)$. Note that the trace $v \mid \Gamma_{D}=0$, for every $v \in W_{\Gamma_{D}}^{1, p}(\Omega)$.

Theorem 3.1 Under the assumptions of Section 2, namely (2.6) and (2.18), suppose the solution map $z \rightarrow w(z)$, associated to the problem (2.3)(2.4), applies $M$ into $W^{l, p}(\Omega)$ and, for some $p>N$ and some constant $\lambda=$ $\lambda\left(M, k, g, \Omega, \Gamma_{D}, p\right)>0$ :

$$
\left\|\nabla w\left(z_{l}\right)-\nabla w\left(z_{2}\right)\right\|_{L^{p(x)}} \leq \lambda\left\|z_{I}-z_{2}\right\|_{C^{D}(\bar{\Omega})} .
$$
that:

Then there exists a unique solution $u$ to (2.I), provided the data are such

$$
\lambda C_{n} e^{-k \eta_{*} / k<1} \text {. }
$$

Proof Recalling (2.5) and (2.7) we have, for $\psi_{i}=\Psi\left(z_{i}\right)$ and $w_{i}=w\left(z_{i}\right)$, $i=1,2$,

$$
\left|\psi_{1}-\psi_{2}\right|=\frac{1}{k}\left|\log w_{1}-\log w_{2}\right| \leq \frac{1}{k} \sup _{w \geq e^{\eta_{*}}}\left|\frac{1}{w}\right|\left|w_{1}-w_{2}\right| .
$$

hence, using (2.15), (3.1) and (3.2) we obtain, for $u_{i}=u\left(z_{i}\right)$,

$$
\begin{gathered}
\left\|u_{1}-u_{2}\right\|_{C^{0}(\bar{\Omega})} \leq\left\|\psi_{1}-\psi_{2}\right\|_{C^{0}(\bar{\Omega})} \leq\left(e^{\left.-k \eta_{*} / k\right)}\left\|w_{1}-w_{2}\right\|_{C^{0}(\bar{\Omega})}\right. \\
\leq\left(e^{\left.-k \eta_{*} / k\right)} C_{p}\left\|\nabla\left(w_{1}-w_{2}\right)\right\|_{L^{p}(\Omega)} \leq \delta\left\|z_{1}-z_{2}\right\|_{C^{0}(\bar{\Omega})},\right.
\end{gathered}
$$

where $\delta \equiv C_{p}\left(e^{-k \eta_{*}} / k\right) \lambda<1$. Therefore $T: z \rightarrow u_{z}$ is a strict contraction in $M$ and the conclusion follows.

We discuss now three cases where the estimate (3.2) holds. First, for $N=1$ it is immediate that (3.2) holds with $p=2$, from the estimate (2.8). We can be more precise on the smallness condition (3.3) if we specify our problem, as in [N], for instance:

$$
\Omega=] 0, \ell\left[, \Gamma_{o}=\Gamma_{D}=\partial \Omega \text { and }-\nu=g(0)<g(\ell)=0=h(0)<h(\ell)=\beta .\right.
$$

In this case, we can compute $\lambda$ in the following way: noting that $-v \leq z_{2} \leq 2 \mu$, we have an "a priori" bound on $\left\|w_{2}^{\prime}\right\|_{\mathrm{L}^{2}(0, \Omega)}$, from

$$
\begin{gathered}
e^{-2 k \mu} \int_{0}^{\ell}\left|w_{2}^{\prime}\right|^{2} d x \leq \int_{0}^{\ell} e^{-k z_{2}}\left|w_{2}^{\prime}\right|^{2} d x=\left(1-e^{-k v}\right) / \ell \int_{0}^{\ell} e^{-k z_{2}} w_{2}^{\prime} d x \\
\leq\left(\left(e^{k v}-1\right) / \sqrt{\ell}\right)\left(\int_{0}^{\ell}\left|w_{2}^{\prime}\right|^{2} d x\right)^{1 / 2}
\end{gathered}
$$


(by using the test function $\left.v(x)=\left(1-e^{-k v}\right) x / \ell+e^{-k \nu}-w_{2}\right)$; which, introduced in (2.9) with $\xi_{1}=e^{-2 k \mu}$, yields (3.3) with $p=2$ and

$$
\lambda=k e^{k\left(4_{\mu}+v\right)}\left(e^{k v}-1\right) / \sqrt{\ell} .
$$

Noting that we can take now $C_{2}=\sqrt{\ell}$ in (3.1) and $\eta_{*}=0$ we may choose $\delta=\left(e^{k v}-1\right) e^{k(4 \mu+v)}$ and we have the following consequence of Theorem 3.1:

Corollary 3.1 In the one dimensional case (3.4), there exists a unique solution of (2.1), provided $\left(e^{k v}-1\right) e^{k(4 \mu+w)}<1$.

Remark 3.1 Note that $\mu$, defined in (2:17), depends on $f$ and on $\beta$. We observe that if, as in the physical case, $f \leq 0$, by Theorem 4:5.4 of [R] applied to (1.11), we may replace $2 \mu$ exactly by $\beta$, yielding, in particular, the " $a$ priori". estimate $-u \leq u \leq \beta$. As a consequence, with the sufficient condition $\left(e^{k v}-1\right) e^{k(2 \beta+u)}<1$, that is to say, for sufficiently small values of the potentials $\beta$ and $v$, we have uniqueness of solutions near the stable equilibrium nul state. Corollary 3.1 yields a much more acurate uniqueness criteria than the previous one of $[\mathrm{N}]$.

For the mixed problem in higher dimensions the estimate (3.2) for $p>N$ is a delicate question. However the extension of Meyers estimate, recently given in $[G]$, to the mixed problem yields an interesting application to the bidimensional case.

Corollary 3.2 Let $N=2$ and suppose that the Lipschitz boundary $\partial \Omega$ is decomposed into $\Gamma_{D}$ and $\partial \Omega \backslash \Gamma_{D}$ with $\bar{\Gamma}_{D} \cap\left(\partial \Omega \backslash \Gamma_{D}\right)$ consisting of a finite number of points. Then, for sufficiently small data, in particular, if the Dirichlet data $g$ has small variation. there exists a unique solution of the quasi-variational problem (2.1).

$*$

Proof Let $\tilde{g} \in \mathrm{W}^{1, p}(\Omega)$, for $p>2$, be an extension of the boundary data. Then the variational solution $w$ of the mixed problem (2.3)-(2.4) may be given by $w=\tilde{w}+e^{-k \hat{g}}$, where $\tilde{w}$ is the unique solution of

$$
\tilde{w} \in \mathrm{W}_{\Gamma_{D}}^{1.2}(\Omega): \int_{\Omega} e^{k z} \nabla \tilde{w} \cdot \nabla v d x=\int_{\Omega} \mathbf{F} \cdot \nabla v d x, \quad \forall v \in \mathrm{W}_{\Gamma_{i}}^{1.2}(\Omega),
$$

with $\mathbf{F}=k e^{k(z-\tilde{g})} \nabla \tilde{g} \in\left[L^{p}(\Omega)\right]^{2}$, for $p>2$ and for each $z \in M$.

Since $z \in M$, from Theorem 1 of [G], there exists a $q, 2<q \leq p$, and a constant $\mathrm{L}_{q}=\mathrm{L}\left(q, \Omega, \Gamma_{p}, \xi_{*}, \xi^{*}\right)>0$, such that,

$$
\left\|\nabla \tilde{w^{\prime}}\right\|_{\mathrm{L}^{q}(\Omega)} \leq \mathrm{L}_{q}\|\mathbf{F}\|_{\mathrm{L}^{q}(\Omega)} \leq k \mathrm{~L}_{q} \sup e^{k(z-\tilde{g})}\|\nabla \tilde{g}\|_{\mathrm{L}^{q}(\Omega)} .
$$


Considering now $w_{1}=w\left(z_{1}\right)$ and $w_{2}=w\left(z_{2}\right)$, we easily see that $\bar{w}=w_{1}-w_{2}$ is also the solution of (3.5), now with $z$ replaced by $z_{1}$ and $\mathbf{F}$ by $\overline{\mathbf{F}}=\left(e^{k z_{2}}-e^{k z_{1}}\right)$ $\nabla w_{2} \in\left[L^{q}(\Omega)\right]^{2}$, by (3.6). Analogously we obtain, for some $r, 2<r \leq q$ :

$$
\begin{aligned}
& \|\nabla \bar{w}\|_{L^{r}(\Omega)} \leq \mathrm{L}_{r}\|\nabla \overline{\mathbf{F}}\|_{\mathrm{L}^{r}(\Omega)} \leq \mathrm{L}_{r}\left\|e^{k=2}-e^{k z_{1}}\right\|_{\mathrm{L}^{x}(\Omega)}\left\|\nabla w_{2}\right\|_{L^{4}(\Omega)} \\
& \leq\left(k^{2} \mathrm{~L}_{q} \mathrm{~L}_{r} \sup _{\Omega} e^{k\left(z_{2}-\tilde{g}\right)}\|\nabla \tilde{g}\|_{\mathrm{L}^{q}(\Omega)} \sup _{z \in M} e^{k z}\right)\left\|z_{1}-z_{2}\right\|_{C^{0}(\bar{\Omega})} .
\end{aligned}
$$

This yields an expression for $\lambda$ in the corresponding estimate (3.2), which implies the conclusion of this Corollary.

Remark 3.2 This existence and uniqueness result holds, in particular, in any domain which boundary is piecewise of class $C^{\prime}$ and whose vertices are not cusps, as for instance, in any polygonal domain. A more restrictive result was presented in [N]; for a rectangular domain, in which Grisvard's results for elliptic equations with mixed boundary conditions yields a $\mathrm{H}^{2}(\Omega) \cap \mathrm{W}^{1 . \infty}(\Omega)$ solutions for (2.3)-(2.4) (see [N] or [R], for references).

Nevertheless if $\partial \Omega \backslash \Gamma_{D}=\varnothing$ in (2.4), the regularity of the Dirichlet problem holds for every $\mathrm{W}_{0}^{1, p}(\Omega)$ and we can state the following result.

Corollary 3.3 For arbitrary dimension $N$, if $\Gamma_{D}=\partial \Omega$ is of class $C^{\prime}$ and (2.6) and (2.18) hold, then there exists an $\varepsilon_{0}>0$, such that, if

$$
\|g\|_{w^{\prime-1 / p, p}}(\partial \Omega) \leq \varepsilon_{0}, \quad \text { for some } p>N,
$$

there exists a unique solution of (2.1).

Proof: Using the $W_{0}^{l, p}(\Omega)$-regularity of the homogeneous Dirichlet problem (3.5) in $\mathrm{H}_{0}^{\prime}(\Omega)$ (see, e.g., Thm. 3:7.2 of [R] and its references) and arguing as in the previous Corollary, we have (3.6) and (3.7) for $q=r=p>N$. Then the conclusion is immediate, by recalling the corresponding dependence of $\lambda$ on $g$ and the condition (3.3).

\section{APPLICATION TO REVERSE BIASED pn-JUNCTIONS}

In this section we extend the existence and uniqueness results for the model problem (2.1) to the following similar quasi-variational inequality correponding to the $p n$-junction model (1.4)-(1.5):

$$
u \in \mathrm{C}(u): \int_{\Omega} \nabla u \cdot \nabla(v-u) d x \geq \int_{\Omega} f(v-u) d x, \quad \forall v \in \mathbf{C}(u)
$$


Here we suppose $\Omega=\Omega_{1} \cup \Gamma \cup \Omega_{2}$ is a Lipschitz domain, such that, each subdomain $\Omega_{1}$ and $\Omega_{2}$ have also Lipschitz boundaries $\partial \Omega_{1}$ and $\partial \Omega_{2}$, with $\Gamma=\partial \Omega_{1} \cap \Omega=\partial \Omega_{2} \cap \Omega \neq \varnothing$. Accordingly, we choose $f=f_{1}$ on $\Omega_{1}$ and $f=f_{2}$ on $\Omega_{2}$ and we define, for each $z \in \mathrm{L}^{\infty}(\Omega)$ and for $\Gamma_{\mathrm{o}} \subset \partial \Omega\left(\Gamma_{\mathrm{o}}\right.$ open, $\left.\Gamma_{0} \neq \varnothing\right)$

$$
\mathrm{C}(z)=\left\{v \in \mathrm{H}^{\prime}(\Omega): v=h \text { on } \Gamma_{0}, v \leq \Phi(z) \text { in } \Omega_{1} \text { and } v \geq \Psi(\mathrm{z}) \text { in } \Omega_{2}\right\} \text {, }
$$

where $z \rightarrow\{\Phi(z), \Psi(z)\}$ is given by (1.8), with the auxiliary mixed problem (1.6)-(1.7), with $z$ instead of $u$ and with

$$
\Gamma_{i} \subset \partial \Omega_{i} \backslash \bar{\Gamma}, \Gamma_{i} \neq \varnothing, g \mid \Gamma_{i}=g_{i} \text {, for } i=1,2 \text { and }-k_{1}=k_{2}=k>0 .
$$

As in the case of only one obstacle, it is necessary to guarantee that $\mathrm{C}(z) \neq \varnothing$, independently of $z$. In a similar way to the assumption (2.18) we shall require the natural compatibility conditions:

$$
\begin{aligned}
& g \geq h \quad \text { on } \quad \Gamma_{1} \cap \Gamma_{0} \quad \text { and } \quad \inf _{\Gamma_{1}} g \geq \underset{\left(\Gamma_{0} \cap \partial \Omega_{1}\right) \backslash \Gamma_{1}}{ } \stackrel{\text { sup }}{ } \\
& g \leq h \text { on } \Gamma_{2} \cap \Gamma_{0} \text { and } \underset{\Gamma_{2}}{\sup } g \leq \underset{\left(\Gamma_{0} \cap \partial \Omega_{2}\right) \backslash \Gamma_{2}}{\inf } h \text {; }
\end{aligned}
$$

and the mathematical expression of the reverse biased conditions:

$$
\sup _{\Gamma_{2}} \mathrm{~g} \leq 0 \leq \inf _{\Gamma_{1}} \mathrm{~g}
$$

Theorem 4.1 Under the preceding assumptions, namely (4.3)-(4.6) and (2.6), the quasi-variational inequality (4.1)-(4.2) has at least one solution, with the regularity

$$
u \in \mathrm{C}^{0} \gamma(\bar{\Omega}) \cap W_{\text {lioc }}^{2 \infty}\left(\Omega_{1} \cup \Omega_{2}\right) \text {, for some } 0<\gamma<1 .
$$

Proof: Since the proof follows the same lines of the one of Theorem 2.1, we only sketch it, refering the necessary changes. We find the solution $u$ as a Schauder fixed point for $T: \mathbf{Z} \ni z \rightarrow u_{z} \in \mathbf{Z}$, defined by the auxiliary problem (2.11) with $\mathbf{K}(z)$, replaced by $\mathbf{C}(z)$; the corresponding double obstacle problem can be reduced, using (2.15), to the simpler problem $(2.12)$ for $\tilde{u}=u_{z}-\xi$, where $\mathbf{K}_{\dot{\psi}}$ is now replaced by $\mathbf{K}_{\tilde{\psi}}^{\tilde{\varphi}}=\left\{v \in \mathbf{H}^{\prime}(\Omega): v=0\right.$ on $\Gamma_{0}, v \leq \tilde{\varphi}$ in $\Omega_{1}$ and $\nu \geq \tilde{\psi}$ in $\left.\Omega_{\dot{2}}\right\}$; the assumptions (4.4) and (4.5) imply $\tilde{\varphi}=\Phi(z)-h \geq 0$ and $\tilde{\psi}=\tilde{\Psi}(z)-h \leq 0$ on $\Gamma_{0}$, independently of $z$; while (4.6), by the maximum principle for (1.6)-(1.7), implies, through (1.8), the conditions $\Phi(z) \geq 0$ in $\Omega_{1}$ and $\Psi(z) \leq 0$ on $\Omega_{2}$; hence $\mathbf{K}_{\vec{\psi}}^{\varphi} \neq \varnothing$ and also $\mathbf{C}(z) \neq \varnothing$ independently of $z$; since the analogous of Proposition 2.1 holds for this double obstacle 
problem, it yields inf $\tilde{\varphi} \Lambda 0 \leq \tilde{u} \leq 0 \vee \sup \tilde{\psi}$ in $\Omega$, which implies the "a priori" estimate for $u_{z}$ :

$$
\xi_{1} \equiv \underset{\Gamma_{1}}{\inf } g-2 \mu \leq u_{z} \leq 2 \mu+\sup _{\Gamma_{2}} g \equiv \xi_{2} \quad \text { in } \Omega
$$

these bounds are, of course, independent of $z$, and they can be used to define $\mathbf{Z}=\left\{v \in \mathrm{C}^{0, \gamma}(\bar{\Omega}): \xi_{1} \leq \nu \leq \xi_{2}\right.$ in $\left.\Omega\right\}$; using the Hölder continuity of each solution and their respective continuous dependence results, the conclusion follows similarly, as well as the local $\mathrm{W}^{2, \infty}$-regularity in $\Omega_{1}$ and in $\Omega_{2}$.

Under similar assumptions, as in Section 3 we can give sufficient conditions on the smallness of the data so that the nonlinear mapping $T$ : $z \rightarrow u_{z}$ is a strict contraction.

Denote by $C_{i}=C_{p}\left(\Omega_{i}\right)$ the corresponding Sobolev constant of (3.1) for functions of $\mathrm{W}_{\Gamma, p}^{1, p}\left(\Omega_{i}\right), p>N(i=1,2)$. Suppose the solution mappings $z \rightarrow w_{i}(z)$, associated with each problem $(1.6)-(1.7)$, apply $\mathbf{Z}$ into $\mathrm{W}^{1, p}\left(\Omega_{i}\right)$ and, analogously to (3.2), we have, for some $\lambda_{i}>0$,

$$
\left\|\nabla w_{i}(z)-\nabla w_{i}(\hat{z})\right\|_{L^{p}\left(\Omega_{i}\right)} \leq \lambda_{i}\|z-\hat{z}\|_{\mathrm{C}^{\circ}\left(\overline{\Omega_{i}}\right),} i=1,2 .
$$

Notice that, if $u=u(z)$ and $\vec{u}=u(\hat{z})$ denote the (unique) solutions of (2.11), respectively, in $\mathbf{C}(z)$ and $\mathbf{C}(\hat{z})$ (with the definition (4.2)), the analogous of $(2.20)$ holds in the following form:

$$
\|u-\hat{u}\|_{C^{o}(\bar{\Omega})} \leq\|\Phi(z)-\Phi(\hat{z})\|_{C^{o}\left(\bar{\Omega}_{1}\right\}} \vee\|\Psi(z)-\Psi(\hat{z})\|_{C^{o}\left(\bar{\Omega}_{2}\right)}
$$

Recalling that $w_{i} \geq \underset{\Gamma_{i}}{\inf } e^{-k_{i} g_{i}}$ and the definitions (1.8), as in the proof of Theorem 3.1, we easily deduce

$$
\|u-\hat{u}\|_{C^{\circ}(\bar{\Omega})} \leq \delta\|z-\hat{z}\|_{C^{0}(\bar{\Omega})}
$$

with $\delta \equiv\left(e^{k \eta_{1}} C_{1} \lambda_{1} / k\right) \vee\left(e^{-k \eta_{2}} C_{2} \lambda_{2} / k\right), \eta_{1}=\sup _{\Gamma} g$ and $\eta_{2}=\underset{\Gamma_{2}}{\inf } \mathrm{g}$.

and the following theorem is then proved.

Therorem 3.2 Under the above assumptions, namely (2.6), (4.3)-(4.6) and (4.8), there exists a unique solution u to (4.1), for sufficient small data, namely, if the following conditions holds

$$
\left(e^{k \eta_{1}} C_{1} \lambda_{1} / k\right) \vee\left(e^{-k \eta_{2}} C_{2} \lambda_{2} / k\right)<1 .
$$


Remark 4.1 As in the Corollary 3.1, for the simple case of one space dimension, with Dirichlet data, the estimate (4.9) can be made more precise by imposing further specifications into our problem.

Remark 4.2 Also the Corollary 3.2 has its natural extension to the corresponding bidimensional model for pn-junctions, by applying the $\mathrm{W}^{\mathrm{l}, q_{-}}$ regularity estimate for mixed problems to both subdomains $\Omega_{1}$ and $\Omega_{2}$, which yields the required estimates (4.8) for some $p>2=N$.

Remark 4.3 The extension of the $N$-dimensional case of Corollary 3.3 is also possible with the following change in the first part of the assumption (4.3): for each $i=1,2$, suppose $\partial \Omega_{i}=\Gamma_{i}$ of class $C^{\prime}$ and $\Gamma_{1} \cap \Gamma_{2}=\Gamma$, with $\Gamma \cap \partial \Omega=\varnothing$; hence the $W^{1, p}$-regulariry holds for each Dirichlet problem in $\Omega_{1}$ and $\Omega_{2}$.

\section{References}

[BC] C. BAIOCCHI and A. CAPElo: Disequazoni variazional e quasi variazionali, Vol. I, II, Quaderni dell' Un. Mat. Ital., Pitagora, Bologna, 1978, English transl. J. Wiley, Chichester-New York, 1984.

[BL] A. BENSOUSSAN and J. L. LIONS: Contrôle impulsionnel et inéquations quasi-variationnelles, 'Dunod, Paris, 1982.

[BCG] F. BREZZZI, A. CAPELO and L. GASTALDI: A singular periturbation analysis of reverse-biased semiconductor diodes, SIAM J. Math. Anal., 20 (1989), 372-387.

[BCM] F. BREZzI, A. CAPELO and L. D. MARINI: Singular perturbation problem in semiconductor devices, Lecture Notes in Math. \#1230, J. P. Hennart, ed., Springer-Verlag, Berlin, New York, 1986, 191-198.

[CF] L. A. CAFFARELLI and A. FRIEDMAN: A singular perturbation problem for semiconductors, Boll. Un. Mat. Ital., B-7 (1987), 409-421.

[HN] C. HuNT and N. R. NASSIF: On a variational inequality and its approximation, in the theory of semiconductors, SIAM J. Numer. Anal., 12 (1975), 938-950.

[Ga] L. GASTALDI: Theoretical results on semiconductor equations, in Computational mathematics and Applications, Proc. 8th France-USSR-Italy Symposium, IAN-CNR, \# 730, Pavia (1989), pp. 207-218.

[G] K. GROGER: $A W^{1, p}$-Estimate for Solutions to Mixed Boundary Value Problems for Second Order Elliptic Differential Equations, Math. Ann. 183 (1989), 679-687.

[KS] D. KinderlehreR and G. STAMPACCHIA: An Introduction to Variational Inequalities and Their Applications, Academic Press, New York, 1980.

[MRS] P. A. MARKowICH, C. A. RINGHOFER and C. SCHMEISER: Semiconducior Equations, Springer-Verlag, Wien, 1990.

[M] U. Mosco: Implicity variational problems and quasi-variational inequalities, in Lect Notes Math. (Springer) \# 543 (1976), 83-156. 
[N] N. NASSiF: On the existence and uniqueness of solutions for a class of a quasi-variational inequalities to solve reverse-biased semiconductor devices. Annali Mat. pura ed. appl. 144 (1986), 95-112.

[NM] N. NASSIF and K. MALLA: Formulation mathématique du comportement de quelques semi-conducteurs au moyen d'une inégalité quasi variationnelle. C. R. Acad. Sc. Paris 294, Ser. I (1982), 79-82.

[R] J. F. RodRigues: Obstacle Problem in Mathematical Physics, NorthHolland, Amsterdam, 1987.

[S] A. SCHMEISER: A singular perturbation analysis of reverse biased $P N$ Junctions, SIAM J. Math. Anal. 21 (1990), 313-326.

CMAF-INIC and Univ. of Lisbon

Av. Prof. Gama Pinto, 2

I699 LISBOA CODEX (PORTUGAL)

Recibido: 20 de febrero de I99| 